\title{
Some Results on Chromatic Polynomials of Hypergraphs
}

\author{
Manfred Walter \\ SAP AG, Dietmar-Hopp-Allee 16, D-69190 Walldorf, Germany \\ Postal Address: Manfred Walter, Schaelzigweg 35, D-68723 Schwetzingen, Germany \\ mwalter-schwetzingen@t-online.de
}

Submitted: Feb 11, 2009; Accepted: Jul 23, 2009; Published: Jul 31, 2009

Mathematics Subject Classifications: 05C15, 05C65

\begin{abstract}
In this paper, chromatic polynomials of (non-uniform) hypercycles, unicyclic hypergraphs, hypercacti and sunflower hypergraphs are presented. The formulae generalize known results for $r$-uniform hypergraphs due to Allagan, Borowiecki/Lazuka, Dohmen and Tomescu.

Furthermore, it is shown that the class of (non-uniform) hypertrees with $m$ edges, where $m_{r}$ edges have size $r, r \geq 2$, is chromatically closed if and only if $m \leq 4$, $m_{2} \geq m-1$.
\end{abstract}

\section{Notation and preliminaries}

Most of the notation concerning graphs and hypergraphs is based on Berge [4].

A hypergraph $\mathcal{H}=(\mathcal{V}, \mathcal{E})$ consists of a finite non-empty set $\mathcal{V}$ of vertices and a family $\mathcal{E}$ of edges which are non-empty subsets of $\mathcal{V}$ of cardinality at least 2. An edge $e$ of cardinality $r(e)$ is called an $r$-edge. $\mathcal{H}$ is $r$-uniform if each edge $e \in \mathcal{E}$ is an $r$-edge. The degree $d_{\mathcal{H}}(v)$ is the number of edges containing the vertex $v$. A vertex $v$ is called pendant if $d_{\mathcal{H}}(v)=1$.

$\mathcal{H}$ is said to be simple if all edges are distinct. $\mathcal{H}$ is is said to be Sperner if no edge is a subset of another edge. Uniform simple hypergraphs are Sperner. Simple 2-uniform hypergraphs are graphs.

A hypergraph $\mathcal{H}^{\prime}=(\mathcal{W}, \mathcal{F})$ with $\mathcal{W} \subseteq \mathcal{V}$ and $\mathcal{F} \subseteq \mathcal{E}$ is called a subhypergraph of $\mathcal{H}$. If $\mathcal{W}=\bigcup_{e \in \mathcal{F}} e$, then the subhypergraph is said to be induced by $\mathcal{F}$, abbreviated by $\mathcal{H}_{\mathcal{F}}$.

The 2-section of a hypergraph $\mathcal{H}=(\mathcal{V}, \mathcal{E})$ is the graph $[\mathcal{H}]_{2}=\left(\mathcal{V},[\mathcal{E}]_{2}\right)$ such that $\{u, v\} \in[\mathcal{E}]_{2}, u \neq v, u, v \in \mathcal{V}$ if and only if $u, v$ are contained in a hyperedge of $\mathcal{H}$.

In a hypergraph $\mathcal{H}=(\mathcal{V}, \mathcal{E})$ an alternating sequence $v_{1}, e_{1}, v_{2}, e_{2}, \ldots, e_{m}, v_{m+1}$, where $v_{i} \neq v_{j}, 1 \leq i<j<m, v_{i}, v_{i+1} \in e_{i}$ is called a chain. Note that repeated edges are 
allowed in a chain. If also $e_{i} \neq e_{j}, 1 \leq i<j \leq m$, we call it a path of length $m$. If $v_{1}=v_{m+1}$, a chain is called cyclic chain, and a path is called cycle. The subhypergraph $\mathcal{C}$ induced by the edge set of a cycle of length $m$ is called a hypercycle, short m-hypercycle. Observe that in case of graphs the notion chain and path, cyclic chain and cycle coincide whereas this is not the case for hypergraphs in general.

A hypergraph $\mathcal{H}$ is said to be connected if for every $v, w \in \mathcal{V}$ there exists a sequence of edges $e_{1}, \ldots, e_{k}, k \geq 1$ such that $v \in e_{1}, w \in e_{k}$ and $e_{i} \cap e_{i+1} \neq \emptyset$, for $1 \leq i<k$. The maximal subhypergraphs which are connected are called components. If a single vertex $v$ or single edge $e$ is a component then $v$ or $e$ is called isolated. We use the abbreviation $\bullet$ for the disjoint union operation, especially of connected components.

According Acharya [1], the relation $\sim$ in $\mathcal{E}$ is an equivalence relation, where $e_{1} \sim e_{2}$ if and only if $e_{1}=e_{2}$ or there exists a cyclic chain containing $e_{1}, e_{2}$. A block of $\mathcal{H}$ is either an isolated vertex/edge or a subhypergraph induced by the edge set of an equivalence class. A block consisting of only one non-isolated edge is called a bridge-block.

Lemma 1.1 ( [1, Theorem 1.1]). Two distinct blocks of a hypergraph have at most one vertex in common.

The block-graph $b c(\mathcal{H})$ of a hypergraph $\mathcal{H}=(\mathcal{V}, \mathcal{E})$ is the bipartite graph created as follows. Take as vertices the blocks of $\mathcal{H}$ and the vertices in $\mathcal{V}$ which are common vertices of two blocks. Two vertices of $b c(\mathcal{H})$ are adjacent if and only if one vertex corresponds to a block $B$ of $\mathcal{H}$ and the other vertex is a common vertex $c \in B$. Observe that in case of graphs we get the block-cutpoint-tree introduced by Harary and Prins [10].

Lemma 1.2 ( $[10$, Theorem 1$])$. If $G$ is a connected graph, then $b c(G)$ is a tree

A hypercycle $\mathcal{C}$ is said to be elementary if $d_{\mathcal{C}}\left(v_{i}\right)=2$ for each $i \in\{1,2, \ldots, m\}$ and each other vertex $u \in \bigcup_{i=1}^{m} e_{i}$ is pendant. This is equivalent to the fact that $\mathcal{C}$ contains only a unique cycle (sequence) up to permutation. A 2-uniform $m$-hypercycle (which is elementary per se) is called m-gon. A hypergraph is linear if any two of its edges do not intersect in more than one vertex. Elementary 2-hypercycles are not linear, whereas elementary $m$-hypercycles, $m \geq 3$, are linear.

A hypertree is a connected hypergraph without cycles. Obviously, a hypertree is linear. A hyperstar is a hypertree where all edges intersect in one vertex. A hyperforest consists of components each of which is a hypertree. A unicyclic hypergraph is a connected hypergraph containing exactly one cycle, i.e. one hypercycle which is elementary.

A hypercactus is a connected hypergraph, where each block is an elementary hypercycle or a bridge-block. Note that this is another approach to generalize the notion of cactus from graphs to hypergraphs as chosen by Sonntag $[14,15]$.

A hypergraph $\mathcal{H}=(\mathcal{V}, \mathcal{E})$ of order $n$ is called a sunflower hypergraph if there exist $\mathcal{X} \subset \mathcal{V},|\mathcal{X}|=q, 1 \leq q<n$ and a partition $\mathcal{V} \backslash \mathcal{X}=\bigcup_{i=1}^{m} \mathcal{Y}_{i}$ such that $\mathcal{E}=\bigcup_{i=1}^{m}\left(\mathcal{X} \bullet \mathcal{Y}_{i}\right)$ Each set $\mathcal{Y}_{i}$ is called a petal, the vertices in $X$ are called seeds. Observe, if $|\mathcal{X}|=1$ then $\mathcal{H}$ is a hyperstar and if $|\mathcal{X}|=2$ then $\mathcal{H}$ is a 2-hypercycle. 
A $\lambda$-coloring of $\mathcal{H}$ is a function $f: \mathcal{V} \rightarrow\{1, \ldots, \lambda\}, \lambda \in \mathbb{N}$, such that for each edge $e \in \mathcal{E}$ there exist $u, v \in e, u \neq v, f(u) \neq f(v)$. The number of $\lambda$-colorings of $\mathcal{H}$ is given by a polynomial $P(\mathcal{H}, \lambda)$ of degree $n$ in $\lambda$, called the chromatic polynomial of $\mathcal{H}$.

Two hypergraphs $\mathcal{H}$ and $\mathcal{H}^{\prime}$ are said to be chromatically equivalent, written $\mathcal{H} \approx \mathcal{H}^{\prime}$, if and only if $\mathrm{P}(\mathcal{H}, \lambda)=\mathrm{P}\left(\mathcal{H}^{\prime}, \lambda\right)$. The equivalence class of $\mathcal{H}$ is abbreviated by $\langle\mathcal{H}\rangle$.

Extending a definition based on Dong, Koh and Teo [8, Chapter 3] from graphs to hypergraphs, a class $\mathcal{H}$ of hypergraphs is called chromatically closed if for any $\mathcal{H} \in \mathcal{H}$ the condition $\langle\mathcal{H}\rangle \subseteq \mathcal{H}$ is satisfied. Let $\mathcal{H}, \mathcal{K}$ be two classes of hypergraphs, then $\mathcal{H}$ is said to be chromatically closed within the class $\mathcal{K}$, if for every $\mathcal{H} \in \mathcal{H} \cap \mathcal{K}$ we have $\langle\mathcal{H}\rangle \cap \mathcal{K} \subseteq \mathcal{H} \cap \mathcal{K}$.

We use the following abbreviations throughout this paper. If $\mathcal{H}$ is isomorphic to $\mathcal{H}^{\prime}$, we write $\mathcal{H} \cong \mathcal{H}^{\prime}$. If $\mathcal{H}=\mathcal{H}_{1} \cup \mathcal{H}_{2}, \mathcal{H}_{1} \cap \mathcal{H}_{2} \cong K_{n}$, we write $\mathcal{H}=\mathcal{H}_{1} \cup_{n} \mathcal{H}_{2}$. $K_{n}$ denotes the complete graph of order $n$, especially $K_{1}$ is an isolated vertex. $\bar{K}_{n}$ denotes the hypergraph consisting of $n \geq 2$ isolated vertices. $\mathcal{S}_{\left.\left(k_{1}\right) r_{1}, \ldots, k_{m}\right) r_{m}}$ denotes a hyperstar with $k_{i} r_{i}$-edges, $i=1, \ldots, m$. $\mathcal{C}_{r_{1}, \ldots, r_{m}}$ denotes the elementary $m$-hypercycle, where $e_{i}$ has size $r_{i}, i=1, \ldots, m$. If $k_{i}$ consecutive edges of the hypercycle have the same size $r_{i}$, we write $\mathcal{C}_{\left(k_{1}\right) r_{1}, \ldots,\left(k_{m}\right) r_{m}}$.

Explicit expressions of chromatic polynomials of hypergraphs were obtained by several authors. In most cases the hypergraphs are assumed to be uniform and linear.

The chromatic polynomials of $r$-uniform hyperforests and $r$-uniform elementary hypercycles were presented by Dohmen [7] and rediscovered by Allagan [3] who used a slightly different notation.

Theorem 1.1 ( [7, Theorem 1.3.2, Theorem 1.3.4], [3, Theorem 1, Theorem 2]). If $\mathcal{H}=(\mathcal{V}, \mathcal{E})$ is an r-uniform hyperforest with $m$ edges and $c$ components, where $r \geq 2$, then

$$
P(\mathcal{H}, \lambda)=\lambda^{c}\left(\lambda^{r-1}-1\right)^{m}
$$

If $\mathcal{H}=(\mathcal{V}, \mathcal{E})$ is an $r$-uniform elementary m-hypercycle, where $r \geq 2, m \geq 3$, then

$$
P(\mathcal{H}, \lambda)=\left(\lambda^{r-1}-1\right)^{m}+(-1)^{m}(\lambda-1)
$$

With the restriction that the hypergraphs are linear, Borowiecki/Lazuka [6] were able to show the converse of (1.1). Combined with the classical result of Read [13] concerning trees, we get

Theorem 1.2 ( [6, Theorem 5], [13, Theorem 13]). If $\mathcal{H}$ is a linear hypergraph and

$$
P(\mathcal{H}, \lambda)=\lambda\left(\lambda^{r-1}-1\right)^{m}, \text { where } r \geq 2, m \geq 1
$$

then $\mathcal{H}$ is an $r$-uniform hypertree with $m$ edges.

Similarly, results of Eisenberg [9], Łazuka [12] for graphs and Borowiecki/Łazuka [6] concerning $r$-uniform unicyclic hypergraphs, $r \geq 3$, can be summarized as follows: 
Theorem 1.3 ( [9], [12, Theorem 2], [6, Theorem 8]). Let $\mathcal{H}$ be a linear hypergraph. $\mathcal{H}$ is an $r$-uniform unicyclic hypergraph with $m+p$ edges and a cycle of length $p$ if and only if

$$
P(\mathcal{H}, \lambda)=\left(\lambda^{r-1}-1\right)^{m+p}+(-1)^{p}(\lambda-1)\left(\lambda^{r-1}-1\right)^{m},
$$

where $r \geq 2, m \geq 0$ and $p \geq 3$.

In parallel Allagan [3, Corollary 3] discovered a slightly different formula for $r$-uniform unicyclic hypergraphs which can be easily transformed into (1.4).

Borowiecki/Lazuka [5, Theorem 5] were the first who studied a class of non-linear uniform hypergraphs which are named sunflower hypergraphs by Tomescu in [17]. In [18] Tomescu gave the following formula of the corresponding chromatic polynomial which we restate in a slightly different notation.

Theorem 1.4 ( $[18$, Lemma 2.1]). Let $\mathcal{S}(m, q, r)$ be an $r$-uniform sunflower hypergraph having $m$ petals and $q$ seeds, where $m \geq 1,1 \leq q \leq r-1$, then

$$
P(\mathcal{S}(m, q, r), \lambda)=\lambda\left(\lambda^{r-q}-1\right)^{m}+\lambda^{(r-q) m}\left(\lambda^{q}-\lambda\right)
$$

The first formulae of chromatic polynomials of non-uniform hypergraphs were mentioned by Allagan [2]. He considered the special case of non-uniform elementary cycles $\mathcal{H}_{m}$ which are constructed from an $m$-gon, $m \geq 3$, by replacing a 2 -edge by a $k+2$-edge, where $k \geq 1$.

Theorem 1.5 ( [2, Theorem 1]). The chromatic polynomial of the hypergraph $\mathcal{H}_{m}, m \geq 3$, has the form:

$$
P\left(\mathcal{H}_{m}, \lambda\right)=(\lambda-1)^{m} \sum_{i=0}^{k} \lambda^{i}+(-1)^{m}(\lambda-1)
$$

Remark 1.1. (1.6) can be restated as follows

$$
P\left(\mathcal{H}_{m}, \lambda\right)=(\lambda-1)^{m-1}\left(\lambda^{k+1}-1\right)+(-1)^{m}(\lambda-1)
$$

Borowiecki/Lazuka [5] extended (1.1) by dropping the uniformity assumption.

Theorem 1.6 ( $\left[5\right.$, Theorem 8]). If $\mathcal{H}=(\mathcal{V}, \mathcal{E})$ is a hyperforest with $m_{r}$ r-edges, where $2 \leq r \leq R$, and c components, then

$$
P(\mathcal{H}, \lambda)=\lambda^{c} \prod_{r=2}^{R}\left(\lambda^{r-1}-1\right)^{m_{r}}
$$

These results suggest to generalize (1.2), (1.4) and (1.5) to non-uniform hypergraphs.

Before we state our results, we remember three useful reduction methods concerning the calculation of chromatic polynomials of hypergraphs.

Given a hypergraph $\mathcal{H}$. If dropping an edge $e \in \mathcal{E}$ yields a hypergraph $\mathcal{H}^{\prime}$ being chromatically equivalent to $\mathcal{H}$, then $e$ is called chromatically inactive. Otherwise, $e$ is said to be chromatically active. Dohmen [7] gave the following lemma: 
Lemma 1.3 ( $\left[7\right.$, Theorem 1.2.1]). A hypergraph $\mathcal{H}$ and the subhypergraph $\mathcal{H}^{\prime}$ which results by dropping all chromatically inactive edges are chromatically equivalent.

The next lemma generalizes Whitney's fundamental reduction theorem. It was already mentioned by Jones [11] in case where the added edge is a 2-edge.

Lemma 1.4. Let $\mathcal{H}=(\mathcal{V}, \mathcal{E})$ be a hypergraph, $X \subseteq \mathcal{V}$ an $r$-set, $r \geq 2$, such that $e \nsubseteq X$ for every $e \in \mathcal{E}$. Let $\mathcal{H}+X$ denote the hypergraph obtained by adding $X$ as a new edge to $\mathcal{E}$ and dropping all chromatically inactive edges. Let $\mathcal{H} . X$ be the hypergraph obtained by contracting all vertices in $X$ to a common vertex $x$ and dropping all chromatically inactive edges. Then

$$
P(\mathcal{H}, \lambda)=P(\mathcal{H}+X, \lambda)+P(\mathcal{H} . X, \lambda)
$$

Proof. We extend the standard proof well-known in the case of graphs.

Let $f$ be a $\lambda$-coloring of $\mathcal{H}$ and $X \subseteq \mathcal{V}$ an $r$-set, $r \geq 2$, such that $e \nsubseteq X$ for every $e \in \mathcal{E}$. Either (i) there exist $u, v \in X$ with $f(u) \neq f(v)$ or (ii) $f(u)=f(v)$ for all $u, v \in X$.

The $\lambda$-colorings of $\mathcal{H}$ for which (i) holds are also $\lambda$-colorings of $\mathcal{H}+X=(\mathcal{V}, \mathcal{E}+X)$ where $\mathcal{E}+X=\mathcal{E} \cup X \backslash \mathcal{E}_{X}$ where $\mathcal{E}_{X}=\{e \in \mathcal{E} \mid X \subset e\}$, and vice versa.

The $\lambda$-colorings of $\mathcal{H}$ for which (ii) holds are also $\lambda$-colorings of $\mathcal{H} . X=(\mathcal{V} . X, \mathcal{E} . X)$ where $\mathcal{V} . X=\mathcal{V} \backslash X \cup\{x\}, \mathcal{E} . X=\{e \backslash X \cup\{x\} \mid e \in \mathcal{E}\}$, and vice versa. Observe that $\mathcal{H} . X$ may contain parallel edges, of which all but one can be dropped as chromatically inactive edges.

Corollary 1.1. Let $\mathcal{H}=(\mathcal{V}, \mathcal{E})$ be a hypergraph. Let $\mathcal{H}-$ e denote the hypergraph obtained by deleting some $e \in \mathcal{E}$ and let $\mathcal{H}$.e be the hypergraph by contracting all vertices in e to a common vertex $x$ and dropping all chromatically inactive edges. Then

$$
P(\mathcal{H}, \lambda)=P(\mathcal{H}-e, \lambda)-P(\mathcal{H} . e, \lambda)
$$

Borowiecki/Eazuka [5] generalized an old result of Read [13].

Lemma 1.5 ( [5, Theorem 6]). If $\mathcal{H}$ is a hypergraph such that $\mathcal{H}=\bigcup_{i=1}^{k} \mathcal{H}_{i}$ for $k \geq 2$, where $\mathcal{H}_{i} \cap \mathcal{H}_{j}=K_{p}$ for $i \neq j$ and $\bigcap_{i=1}^{k} \mathcal{H}_{i}=K_{p}$, then

$$
P(\mathcal{H}, \lambda)=P\left(K_{p}, \lambda\right)^{1-k} \prod_{i=1}^{k} P\left(\mathcal{H}_{i}, \lambda\right) .
$$

\section{The chromatic polynomials of non-uniform hyper- graphs}

Our first generalization concerns non-uniform elementary hypercycles. Note, that elementary 2-hypercycles are not linear whereas elementary $m$-hypercycles, $m \geq 3$, are linear. 
Theorem 2.1. If $\mathcal{C}=(\mathcal{V}, \mathcal{E})$ is an elementary $m$-hypercycle having $m_{r} r$-edges, where $2 \leq r \leq R$, then

$$
P(\mathcal{C}, \lambda)=\prod_{r=2}^{R}\left(\lambda^{r-1}-1\right)^{m_{r}}+(-1)^{m}(\lambda-1)
$$

Our second generalization concerns non-uniform hypercacti.

Theorem 2.2. Let $\mathcal{H}=(\mathcal{V}, \mathcal{E})$ be a hypercactus with

(1) $k$ elementary $p_{i}$-hypercycles $\mathcal{C}_{i}=\left(\mathcal{W}_{i}, \mathcal{F}_{i}\right), i=1, \ldots, k$, having $p_{i r}$ r-edges, where $2 \leq r \leq R$

(2) $m_{r}$ bridge-blocks of size $r, 2 \leq r \leq R$.

Then

$$
P(\mathcal{H}, \lambda)=\frac{1}{\lambda^{k-1}} \prod_{r=2}^{R}\left(\lambda^{r-1}-1\right)^{m_{r}} \prod_{i=1}^{k}\left[\prod_{r=2}^{R}\left(\lambda^{r-1}-1\right)^{p_{i r}}+(-1)^{p_{i}}(\lambda-1)\right]
$$

By converting (2.2), we get the following generalization of Theorem 1.3 concerning non-uniform unicyclic hypergraphs.

Corollary 2.1. Let $\mathcal{H}=(\mathcal{V}, \mathcal{E})$ be a connected unicyclic hypergraph containing a p-hypercycle $\mathcal{C}=(\mathcal{W}, \mathcal{F})$ with $p_{r} r$-edges and containing $m_{r}$ bridge-blocks of size $r$, where $2 \leq r \leq R$, then

$$
P(\mathcal{H}, \lambda)=\prod_{r=2}^{R}\left(\lambda^{r-1}-1\right)^{m_{r}+p_{r}}+(-1)^{p}(\lambda-1) \prod_{r=2}^{R}\left(\lambda^{r-1}-1\right)^{m_{r}}
$$

Our third generalization concerns non-uniform sunflower hypergraphs.

Theorem 2.3. Let $\mathcal{S}$ be a sunflower hypergraph of order $n$ containing $m_{r} r$-edges and $q$ seeds, where $q+1 \leq r \leq R$, then

$$
P(\mathcal{S}, \lambda)=\lambda\left[\lambda^{n-1}-\lambda^{n-q}+\prod_{r=q+1}^{R}\left(\lambda^{r-q}-1\right)^{m_{r}}\right]
$$

Especially in case of uniform hypergraphs we get an alternative expression of Theorem 1.4:

Corollary 2.2. If $\mathcal{H}$ is an $r$-uniform sunflower hypergraph of order $n$ and $q$ seeds, then

$$
P(\mathcal{H}, \lambda)=\lambda\left[\lambda^{n-1}-\lambda^{n-q}+\left(\lambda^{r-q}-1\right)^{m}\right]
$$


Remark 2.1. The proofs of Theorem 2.1, Theorem 2.2 and Theorem 2.3 are based on the fact that the chromatic polynomials can be restated as follows

$$
\begin{aligned}
P(\mathcal{H}, \lambda)= & \lambda^{c} \prod_{x \in \mathcal{E}}\left(\lambda^{r(x)-1}-1\right) \\
P(\mathcal{C}, \lambda)= & \prod_{x \in \mathcal{E}}\left(\lambda^{r(x)-1}-1\right)+(-1)^{m}(\lambda-1) \\
P(\mathcal{H}, \lambda)= & \frac{1}{\lambda^{|I|-1}} \prod_{x \in \mathcal{E} \backslash \mathcal{F}}\left(\lambda^{r(x)-1}-1\right) \prod_{i \in I}\left[\prod_{x \in \mathcal{F}_{i}}\left(\lambda^{r(x)-1}-1\right)+(-1)^{p_{i}}(\lambda-1)\right] \\
& \text { where } \mathcal{F}=\bigcup \bigcup_{i \in I} \mathcal{F}_{i}, I=\{1, \ldots, k\} \\
P(\mathcal{H}, \lambda)= & \prod_{x \in \mathcal{E}}\left(\lambda^{r(x)-1}-1\right)+(-1)^{p}(\lambda-1) \prod_{x \in \mathcal{E} \backslash \mathcal{F}}\left(\lambda^{r(x)-1}-1\right) \\
P(\mathcal{H}, \lambda)= & \lambda\left[\lambda^{n-1}-\lambda^{n-q}+\prod_{x \in \mathcal{E}}\left(\lambda^{r(x)-q}-1\right)\right]
\end{aligned}
$$

Proof of Theorem 2.1. We use induction on the sum $s(\mathcal{C})$ of the edge cardinalities of the elementary $m$-hypercycle $\mathcal{C}$.

The induction starts for each $m$ separately.

For $m=2$, the elementary $m$-hypercycle $\mathcal{C}$ with minimum $s(\mathcal{C})$ consists of two 3 -edges $e, f$, which intersect in exactly two vertices $u_{1}, u_{2}$. Let $v \in e \backslash f$. Replacing the edge $e$ by a 2 -edge $k=\left\{u_{1}, v\right\}$ yields the hypergraph $\mathcal{C}+k$ which is obviously a hypertree with a 3-edge and a 2-edge. Contracting the vertices $u, v$ yields the hypergraph $\mathcal{C} . k$, where $e$ shrinks to the 2-edge $\left\{u_{1}, u_{2}\right\} \subset f$. Therefore $f$ is chromatically inactive in $\mathcal{C}$. $k$ and can be dropped. The resulting chromatically equivalent Sperner hypergraph is isomorphic to $K_{1} \bullet K_{2}$.

By Lemma 1.4 and (2.6), we have

$$
P(\mathcal{C}, \lambda)=\lambda(\lambda-1)\left(\lambda^{2}-1\right)+\lambda^{2}(\lambda-1)=\left(\lambda^{2}-1\right)^{2}+(-1)^{2}(\lambda-1)
$$

This proves the assertion.

For $m \geq 3$ the elementary $m$-hypercycle with minimal $s(\mathcal{C})$ is the $m$-gon. Hence, (2.1) is the well-known formula

$$
P(\mathcal{C}, \lambda)=(\lambda-1)^{m}+(-1)^{m}(\lambda-1) .
$$

The induction step can be made for all $m \geq 2$ simultaneously.

Choose an edge $e$ of the elementary cycle $\mathcal{C}$ with maximal cardinality. If $m=2$, then $r(e) \geq 4$, if $m \geq 3$, then $r(e) \geq 3$. Let $f$ be the predecessor edge in the cycle sequence. Let $u \in e \cap f$ and $v \in e \backslash f$. We create the two hypergraphs $\mathcal{C}+k$ and $\mathcal{C}$. $k$ as follows. We add the 2-edge $k=\{u, v\}$ and shrink the edge $e$ to the edge $e^{\prime}$ by identifying $u, v \cdot e^{\prime}$ remains chromatically active in $\mathcal{C} . k$. 
Obviously, $\mathcal{C}+k$ is a hyperforest and has $r(e)-2$ components where $r(e)-3$ of these are isolated vertices. $\mathcal{C} . k$ is an elementary $m$-hypercycle where $e$ is replaced by $e^{\prime}$ with size $r\left(e^{\prime}\right)=r(e)-1$. Observe that $\mathcal{C}, \mathcal{C}+k$ and $\mathcal{C} . k$ have the same number of edges $m$.

Since $\mathrm{s}(\mathcal{C} . k)=\mathrm{s}(\mathcal{C})-1$, we can apply the induction hypothesis. By (1.9), (2.6) and (2.7), we have

$$
\begin{aligned}
P(\mathcal{C}, \lambda)= & \lambda^{r(e)-2}(\lambda-1) \prod_{g \in \mathcal{E}, g \neq e}\left(\lambda^{r(g)-1}-1\right) \\
& \quad+\left(\lambda^{r\left(e^{\prime}\right)-1}-1\right) \prod_{x \in \mathcal{E}, x \neq e^{\prime}}\left(\lambda^{r(x)-1}-1\right)+(-1)^{m}(\lambda-1) \\
= & \lambda^{r(e)-2}(\lambda-1) \prod_{x \in \mathcal{E}, x \neq e}\left(\lambda^{r(x)-1}-1\right) \\
& \quad+\left(\lambda^{r(e)-2}-1\right) \prod_{x \in \mathcal{E}, x \neq e}\left(\lambda^{r(x)-1}-1\right)+(-1)^{m}(\lambda-1) \\
= & {\left[\lambda^{r(e)-2}(\lambda-1)+\lambda^{r(e)-2}-1\right] \prod_{x \in \mathcal{E}, x \neq e}\left(\lambda^{r(x)-1}-1\right)+(-1)^{m}(\lambda-1) } \\
= & \left(\lambda^{r(e)-1}-1\right) \prod_{x \in \mathcal{E}, x \neq e}\left(\lambda^{r(x)-1}-1\right)+(-1)^{m}(\lambda-1) \\
= & \prod_{x \in \mathcal{E}}\left(\lambda^{r(x)-1}-1\right)+(-1)^{m}(\lambda-1)
\end{aligned}
$$

To simplify the proof of Theorem 2.2 we extend Lemma 1.2 to hypergraphs.

Lemma 2.1. The block-graph bc $(\mathcal{H})$ of a connected hypergraph $\mathcal{H}$ is a tree.

Proof. If $\mathcal{H}$ is a graph, we have nothing to show.

If $\mathcal{H}$ is not a graph, we show that $b c(\mathcal{H}) \cong b c\left([\mathcal{H}]_{2}\right)$. Then Lemma 1.2 completes the proof.

We have to verify that $e, f \in \mathcal{E}$ are in the same block of $\mathcal{H}$ if and only if $e^{\prime}, f^{\prime} \in \mathcal{E}_{2}$ are in the same block of $[\mathcal{H}]_{2}$ for all $e^{\prime} \subseteq e, f^{\prime} \subseteq f$. This implies also that the common vertices of the blocks of $\mathcal{H}$ and $[\mathcal{H}]_{2}$ coincide.

Let $e^{\prime} \subseteq e, f^{\prime} \subseteq f, e^{\prime} \neq f^{\prime}$ be in the same block of $[\mathcal{H}]_{2}$. Then $[\mathcal{H}]_{2}$ contains a cycle $v_{1}, e_{1}^{\prime}, \ldots, e^{\prime}, \ldots, f^{\prime}, \ldots, e_{m}^{\prime}, v_{m+1}, v_{i} \neq v_{j}, 1 \leq i<j<m, v_{1}=v_{m+1}$. We replace every edge $x^{\prime} \in[\mathcal{E}]_{2}$ in this cycle by the corresponding edge $x \in \mathcal{E}, x^{\prime} \subseteq x$. The result is a cycle in $\mathcal{H}$ which contains $e, f$.

Conversely, let $e^{\prime} \subseteq e, f^{\prime} \subseteq f$, where $e, f$ are in the same block of $\mathcal{H}$. Then there exists a cyclic chain $u_{1}, e_{1}, \ldots, e_{n}, u_{n+1}, u_{i} \neq u_{j}, 1 \leq i<j<n, u_{1}=u_{n+1}$, where w.l.o.g. $e_{k}=e, e_{l}=f$ with $1 \leq k<l \leq n$. Replace $e_{i}$ by the 2-edge $\left\{u_{i}, u_{i+1}\right\}$, $i=1, \ldots, n$. If $e^{\prime}=\left\{u_{i}, u_{i+1}\right\}$ and $f^{\prime}=\left\{u_{j}, u_{j+1}\right\}$, we are finished. Assume that $e^{\prime}=\{u, v\}, u, v \in e$, with $\{u, v\} \neq\left\{u_{i}, u_{i+1}\right\}$ for all $i=1, \ldots, n$. Then the cycle $u,\{u, v\}, v,\left\{v, u_{i}\right\}, u_{i},\left\{u_{i}, u_{i+1}\right\}, u_{i+1}\left\{u_{i+1}, u\right\}, u$ exists because each substituted 2-edge 
exists by the definition of $[\mathcal{H}]_{2}$. It follows that $e^{\prime},\left\{u_{i}, u_{i+1}\right\}$ and $\left\{u_{j}, u_{j+1}\right\}$ are in the same block of $[\mathcal{H}]_{2}$. We apply the same argument to $f^{\prime}$ to complete the proof.

Proof of Theorem 2.2. We use induction on the number $b$ of blocks.

If $b=1$, then $\mathcal{H}$ is either a bridge-block or consists of an elementary hypercycle. The evaluation of (2.2) yields either (1.1) or (2.1).

If $b \geq 2, b c(\mathcal{H})$ is a tree by Lemma 2.1. Therefore, we can split $\mathcal{H}=\mathcal{Y} \cup_{1} \mathcal{Z}$, where $\mathcal{Y}, \mathcal{Z}$ are hypercacti. Obviously, the hypercycles and bridge-blocks of $\mathcal{H}$ are divided in those of $\mathcal{Y}$ and $\mathcal{Z}$, i.e. $\mathcal{F}_{\mathcal{Y}}=\mathcal{F} \cap \mathcal{E}_{\mathcal{Y}}$ and $\mathcal{F}_{\mathcal{Z}}=\mathcal{F} \cap \mathcal{E}_{\mathcal{Z}}$, where $\mathcal{E}_{\mathcal{Y}}, \mathcal{E}_{\mathcal{Z}}$ are the edge sets of $\mathcal{Y}, \mathcal{Z}$. Hence we can use the induction hypothesis and (1.11).

$$
\begin{aligned}
& P(\mathcal{H}, \lambda)= \frac{1}{\lambda} P(\mathcal{Y}, \lambda) P(\mathcal{Z}, \lambda) \\
&= \frac{1}{\lambda}\left(\frac{1}{\lambda^{\left|I_{\mathcal{Y}}\right|-1}} \prod_{x \in \mathcal{E}_{\mathcal{Y}} \backslash \mathcal{F}_{\mathcal{Y}}}\left(\lambda^{r(x)-1}-1\right) \prod_{i \in I_{\mathcal{Y}}}\left[\prod_{x \in \mathcal{F}_{i}}\left(\lambda^{r(x)-1}-1\right)+(-1)^{p_{i}}(\lambda-1)\right]\right) \\
&\left(\frac{1}{\lambda^{\left|I_{\mathcal{Z}}\right|-1}} \prod_{x \in \mathcal{E}_{\mathcal{Z}} \backslash \mathcal{F}_{\mathcal{Z}}}\left(\lambda^{r(x)-1}-1\right) \prod_{i \in I_{\mathcal{Z}}}\left[\prod_{x \in \mathcal{F}_{i}}\left(\lambda^{r(x)-1}-1\right)+(-1)^{p_{i}}(\lambda-1)\right]\right) \\
&= \frac{1}{\lambda^{\left|\mathcal{I}_{\mathcal{Y}}\right|+\left|I_{\mathcal{Z}}\right|-1}} \prod_{x \in\left(\mathcal{E}_{\mathcal{Y}} \backslash \mathcal{F}_{\mathcal{Y}}\right) \cup\left(\mathcal{E}_{\mathcal{Z}} \backslash \mathcal{F}_{\mathcal{Z}}\right)}\left(\lambda^{r(x)-1}-1\right) \\
& \quad \times \prod_{i \in I_{\mathcal{Y}} \cup I_{\mathcal{Z}}}\left[\prod_{x \in \mathcal{F}_{i}}\left(\lambda^{r(x)-1}-1\right)+(-1)^{p_{i}}(\lambda-1)\right] \\
&=\frac{1}{\lambda^{|I|-1}} \prod_{x \in \mathcal{E} \backslash \mathcal{F}}\left(\lambda^{r(x)-1}-1\right) \prod_{i \in I}\left[\prod_{x \in \mathcal{F}_{i}}\left(\lambda^{r(x)-1}-1\right)+(-1)^{p_{i}}(\lambda-1)\right]
\end{aligned}
$$

Proof of Theorem 2.3. Assume first that the sunflower hypergraph $\mathcal{S}$ has only one petal, i.e. $\mathcal{S}$ consists of one edge of size $q+1 \leq r \leq R$. Then by (2.4)

$$
P(\mathcal{S}, \lambda)=\lambda\left[\lambda^{r-1}-\lambda^{r-q}+\left(\lambda^{r-q}-1\right)\right]=\lambda\left(\lambda^{r-1}-1\right)
$$

For the remaining cases, we use induction on $n-q$. The case $n-q=1$ was just verified.

Let $u \in Y, Y$ be a petal of $\mathcal{S}$ and $v$ be a seed. Add the edge $k=\{u, v\}$ to $\mathcal{S}$. Then the edge $e=X \cup Y$ becomes chromatically inactive. We consider two cases.

Case 1: The petal $Y$ can be chosen to have size 1 .

Then $\mathcal{S}+k \cong K_{2} \cup_{1} \mathcal{U}$, where $\mathcal{U}$ is the sunflower hypergraph induced by $\mathcal{E} \backslash e$, with $e=X \cup Y$. We contract $k$ and drop all chromatically inactive edges. We receive the 
Sperner hypergraph $\mathcal{S} . k=\bar{K}_{\sum_{x \in \mathcal{E} \backslash e}(r(x)-q)} \bullet \mathcal{H}_{\{X\}}$ because $e$ shrinks to $X$. By Lemma 1.4 and $(2.10)$

$$
\begin{aligned}
P(\mathcal{S}, \lambda)= & (\lambda-1) \lambda\left[\lambda^{n-2}-\lambda^{n-q-1}+\prod_{x \in \mathcal{E} \backslash e}\left(\lambda^{r(x)-q}-1\right)\right]+\lambda\left(\lambda^{q-1}-1\right) \lambda^{\sum_{x \in \mathcal{E} \backslash e}(r(x)-q)} \\
& \text { by induction hypothesis } \\
=\lambda & {\left[(\lambda-1) \lambda^{n-2}-(\lambda-1) \lambda^{n-q-1}+(\lambda-1) \prod_{x \in \mathcal{E} \backslash e}\left(\lambda^{r(x)-q}-1\right)+\left(\lambda^{q-1}-1\right) \lambda^{n-q-1}\right] } \\
& \text { because } \sum_{x \in \mathcal{E} \backslash e}(r(x)-q)=n-q-1 \\
=\lambda & {\left[\lambda^{n-1}-\lambda^{n-q}+\prod_{x \in \mathcal{E}}\left(\lambda^{r(x)-q}-1\right)\right] } \\
& \text { because } \lambda^{r(e)-q}=\lambda
\end{aligned}
$$

Case 2: All petals, especially $Y$, have size greater 1 .

Then $\mathcal{S}+k \cong \bar{K}_{r(e)-q-1} \cup\left(K_{2} \cup_{1} \mathcal{U}\right)$, where $\mathcal{U}$ is the sunflower hypergraph induced by $\mathcal{E} \backslash e$, having $n-r(e)+q$ vertices. $\mathcal{S} . k$ is the sunflower hypergraph of order $n-1$ which is induced by $\mathcal{E} \backslash e \cup e^{\prime}$, where $e^{\prime}=X \cup Y^{\prime}, Y^{\prime}=Y \backslash\{u\}$ is a petal. All other petals remain chromatically active in $\mathcal{S}$.k. Thus,

$$
\begin{aligned}
P(\mathcal{S}, \lambda)= & \lambda(\lambda-1) \lambda^{r(e)-q-1}\left[\lambda^{n-r(e)+q-1}-\lambda^{n-r(e)-1}+\prod_{x \in \mathcal{E} \backslash e}\left(\lambda^{r(x)-q}-1\right)\right] \\
+ & \lambda\left[\lambda^{n-2}-\lambda^{n-q-1}+\left(\lambda^{r\left(e^{\prime}\right)-q}-1\right) \prod_{x \in \mathcal{E} \backslash e^{\prime}}\left(\lambda^{r(x)-q}-1\right)\right] \\
& \text { by induction hypothesis } \\
= & \lambda\left[\lambda^{n-1}-\lambda^{n-q}-\lambda^{n-2}+\lambda^{n-q-1}+(\lambda-1) \lambda^{r(e)-q-1} \prod_{x \in \mathcal{E} \backslash e}\left(\lambda^{r(x)-q}-1\right)\right. \\
+ & \left.\lambda^{n-2}-\lambda^{n-q-1}+\left(\lambda^{r(e)-q-1}-1\right) \prod_{x \in \mathcal{E} \backslash e}\left(\lambda^{r(x)-q}-1\right)\right] \\
= & \lambda\left[\lambda^{n-1}-\lambda^{n-q}+\prod_{x \in \mathcal{E}}\left(\lambda^{r(x)-q}-1\right)\right]
\end{aligned}
$$




\section{Chromaticity of hypertrees}

The fact that trees are chromatically closed within the class of graphs can be extended to the case of $r$-uniform hypertrees, $r \geq 2$, by use of the following lemma due to Tomescu [16] in combination with Theorem 1.2 and (1.1).

Lemma 3.1 ( [16, Lemma 3.1]). If simple $r$-uniform hypergraphs $\mathcal{H}$ and $\mathcal{G}$ are chromatically equivalent and $\mathcal{H}$ is linear then $\mathcal{G}$ is linear too.

Theorem 3.1. The class of $r$-uniform hypertrees is chromatically closed within the class of $r$-uniform hypergraphs, where $r \geq 2$.

Borowiecki/Łazuka already mentioned in [6], without giving concrete examples, that the class of $r$-uniform hypertrees might not be chromatically closed in general. The following theorem shows that this is indeed true except for a few cases.

Theorem 3.2. The class $\mathcal{T}$ of hypertrees with $m$ edges, where $m_{r}$ edges have size $r, r \geq 2$, is chromatically closed if and only if $m \leq 4, m_{2} \geq m-1$.

To prove this, we use some lemmas concerning the coefficients of the chromatic polynomial of a hypergraph $\mathcal{H}$ of order $n$ expressed in the standard form

$$
P(\mathcal{H}, \lambda)=\sum_{i=0}^{n} a_{i} \lambda^{n-i}
$$

Borowiecki/Łazuka [6] showed

Lemma 3.2 ( $[6$, Lemma 1]). Let $\mathcal{H}$ be a hypergraph of order $n$ and the chromatic polynomial expressed by (3.1). If $a_{n-1} \neq 0$ then $\mathcal{H}$ is connected.

Dohmen [7] showed

Lemma 3.3 ( $\left[7\right.$, Theorem 1.4.1]). Let $\mathcal{H}$ be a hypergraph of order $n$ having $m_{r}$ edges of minimal size $r$, where $2 \leq r \leq n$ and the chromatic polynomial expressed by (3.1). Then $a_{k}=0, k=1, \ldots, r-2$ and $a_{r-1}=-m_{r}$.

Proof of Theorem 3.2. We show first that the class of all hypertrees is chromatically closed if $m \leq 4, m_{2} \geq m-1$. It suffices to consider only hypertrees having exactly four edges by the following reason. If a hypertree $\mathcal{T}$ with $m \leq 3$ edges would be chromatically equivalent to hypergraph $\mathcal{H}$ which is not a tree then $\mathcal{H} \cup_{1} \mathcal{S}_{(4-m) 2}$ would be chromatically equivalent to a hypertree with four edges.

Assume there exists a Sperner hypergraph $\mathcal{H}$ which is chromatically equivalent to a hypertree with four edges and at most one $r$-edge, $r \geq 3$. Obviously, $\mathcal{H}$ is connected by Lemma 3.2 and if $\mathcal{H}$ has the same number of $k$-edges as $\mathcal{T}$ then it is hypertree. We therefore inspect the number $m_{k}$ of $k$-edges of $\mathcal{H}, k=2, \ldots, r+3$.

Clearly $m_{r+3}=0$, because no chromatically active $r+3$-edge can exist. Furthermore Lemma 3.3 implies that $\mathcal{H}$ has the same number of 2-edges as $\mathcal{T}$, i.e. $m_{2}=3$, if $r \geq 3$, and $m_{2}=4$, if $r=2$. 
To verify the remaining cases $m_{k}, 2<k \leq r+2$, observe that if $m_{k} \neq 0$ then $\mathcal{H}$ contains a spanning hypergraph with one $k$-edge and all 2-edges. This hypergraph is either a forest or one of the hypergraphs $\mathcal{H}_{i}, i=1, \ldots, 6$ in Figure 1.
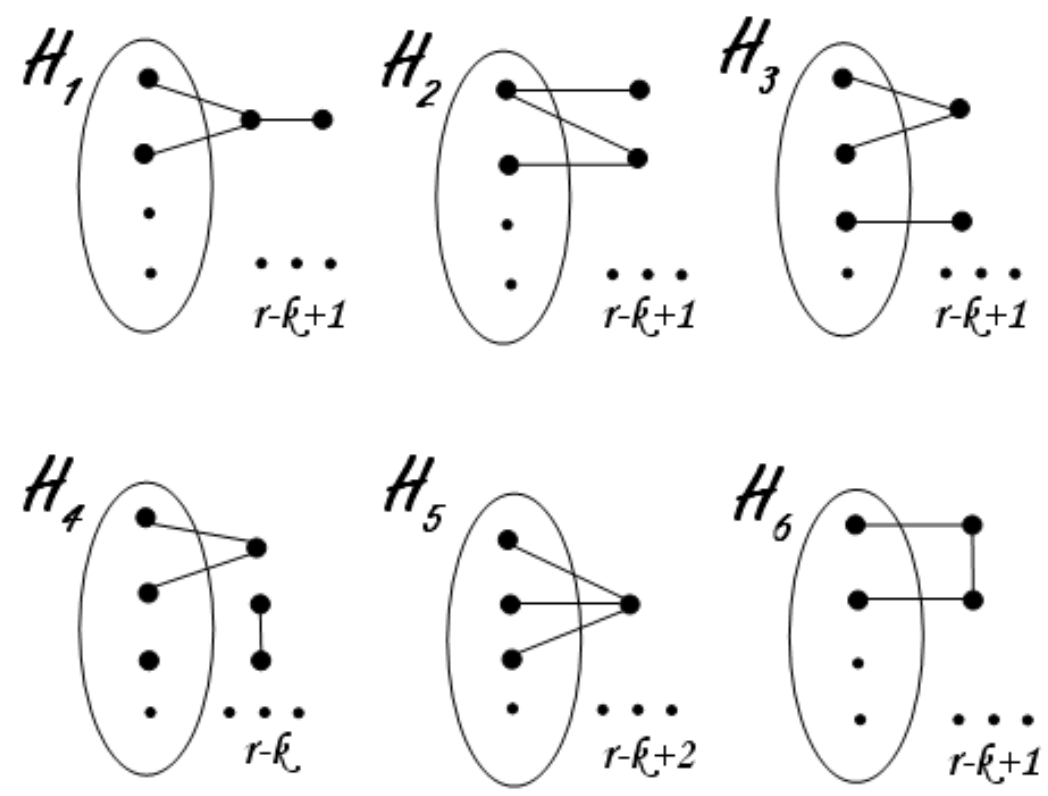

Figure 1

First $m_{r+2}=0$. Otherwise, assume there exists an $r+2$-edge. Since $\lambda-2 \nmid P(\mathcal{H}, \lambda)$ we conclude that $K_{3} \nsubseteq \mathcal{H}$. The fact that $\mathcal{H}$ is Sperner implies that $\mathcal{H} \cong \mathcal{H}_{5}$, where no isolated vertices exist.

We delete/contract the $r+2$-edge. By (1.10)

$$
\begin{aligned}
P(\mathcal{H}, \lambda) & =\lambda^{r}(\lambda-1)^{3}-\lambda(\lambda-1)=\lambda\left(\lambda^{r-1}-1\right)(\lambda-1)^{3}+\lambda(\lambda-1)^{3}-\lambda(\lambda-1) \\
& \neq P(\mathcal{T}, \lambda)
\end{aligned}
$$

Next, we show that $m_{k}=0,2<k<r$. This is done by comparing $P(\mathcal{H}, \lambda)$ and $P(\mathcal{T}, \lambda)$ for $\lambda \in \mathbb{N}$.

Assume that $\mathcal{H}$ contains a spanning hyperforest $\mathcal{F}$ with all the 2-edges and one $k$-edge, $2<k \leq r$. By (1.8) we have

$$
\begin{aligned}
P(\mathcal{H}, \lambda) & \leq P(\mathcal{F}, \lambda)=\lambda^{r-k+1}\left(\lambda^{k-1}-1\right)(\lambda-1)^{3} \\
& =\lambda\left(\lambda^{r-1}-1\right)(\lambda-1)^{3}-\lambda\left(\lambda^{r-k}-1\right)(\lambda-1)^{3} \leq P(\mathcal{T}, \lambda)
\end{aligned}
$$

Only in case $k=r$ equality holds, i.e. $\mathcal{H} \approx \mathcal{F} \approx \mathcal{T}$.

Assume next that $\mathcal{H}_{i} \subseteq \mathcal{H}$ for some $i=1, \ldots, 6$ and $k \leq r$. 
If $\mathcal{H}_{i} \subseteq \mathcal{H}$ for $i=1, \ldots, 4$, we apply (2.1) and (1.11).

$$
\begin{aligned}
P(\mathcal{H}, \lambda) & \leq P\left(\mathcal{H}_{i}, \lambda\right)=\lambda^{r-k+1}(\lambda-1)\left[\left(\lambda^{k-1}-1\right)(\lambda-1)^{2}-(\lambda-1)\right] \\
& =\lambda\left(\lambda^{r-1}-1\right)(\lambda-1)^{3}-\lambda(\lambda-1)^{2}\left(\lambda^{r-k+1}-\lambda+1\right) \\
& <P(\mathcal{T}, \lambda), \text { because of } k \leq r .
\end{aligned}
$$

If $\mathcal{H}_{5} \subseteq \mathcal{H}$ we delete/contract the $k$-edge and apply (1.10), (1.11) and (2.1).

$$
\begin{aligned}
P(\mathcal{H}, \lambda) & \leq P\left(\mathcal{H}_{5}, \lambda\right)=\lambda^{r}(\lambda-1)^{3}-\lambda^{r-k+3}(\lambda-1) \\
& =\lambda\left(\lambda^{r-1}-1\right)(\lambda-1)^{3}-\lambda(\lambda-1)\left[\lambda^{r-k+2}-\lambda^{2}+2 \lambda-1\right] \\
& <P(\mathcal{T}, \lambda), \text { because of } k \leq r .
\end{aligned}
$$

If $\mathcal{H}_{6} \subseteq \mathcal{H}$ and $k<r$, we apply (1.11) and (2.1).

$$
\begin{aligned}
P(\mathcal{H}, \lambda) & \leq P\left(\mathcal{H}_{6}, \lambda\right)=\lambda^{r-k+1}\left[\left(\lambda^{k-1}-1\right)(\lambda-1)^{3}+(\lambda-1)\right] \\
& =\lambda\left(\lambda^{r-1}-1\right)(\lambda-1)^{3}-\lambda(\lambda-1)\left(\lambda^{r-k+2}-2 \lambda^{r-k+1}-\lambda^{2}+2 \lambda-1\right) \\
& <P(\mathcal{T}, \lambda), \text { because of } k<r .
\end{aligned}
$$

Consider $\mathcal{H}_{6} \subseteq \mathcal{H}$ and $k=r . \mathcal{H} \cong \mathcal{H}_{6}$ is impossible because (1.11) and (2.1) imply

$$
P\left(\mathcal{H}_{6}, \lambda\right)=\lambda\left[\left(\lambda^{r-1}-1\right)(\lambda-1)^{3}+(\lambda-1)\right]>P(\mathcal{T}, \lambda)
$$

Therefore $\mathcal{H}$ must contain additional edges, each of size $r$ or size $r+1$. If we delete these edges in an arbitrary sequence until $\mathcal{H}_{6}$ remains, the order of the hypergraphs resulting from the contraction is always at least 3. Applying (1.10) repeatedly subtracts from $P\left(\mathcal{H}_{6}, \lambda\right)$ a polynomial of at least degree 3 . Hence $P(\mathcal{H}, \lambda)<P(\mathcal{T}, \lambda)$.

In summary, we get that $m_{k}=0$ for $2<k<r$ and that if $\mathcal{H}$ contains an $r$-edge then $\mathcal{H}$ is a tree.

It remains to exclude the case that a hypergraph containing only $r+1$-edges besides the 2-edges is chromatically equivalent to $\mathcal{T}$. Obviously, $\mathcal{H}$ cannot contain a subhypergraph isomorphic to $\mathcal{H}_{4}$.

If $\mathcal{H} \cong \mathcal{H}_{i}, i=1, \ldots, 3$, we apply (2.1) and (1.11)

$$
\begin{aligned}
P\left(\mathcal{H}_{i}, \lambda\right) & =(\lambda-1)\left[\left(\lambda^{r}-1\right)(\lambda-1)^{2}-(\lambda-1)\right] \\
& =\lambda\left(\lambda^{r-1}-1\right)(\lambda-1)^{3}+\lambda(\lambda-1)^{2}(\lambda-2)>P(\mathcal{T}, \lambda)
\end{aligned}
$$

If $\mathcal{H} \cong \mathcal{H}_{5}$, we apply (1.10), (2.1) and (1.11)

$$
\begin{aligned}
P\left(\mathcal{H}_{5}, \lambda\right) & =\lambda^{r}(\lambda-1)^{3}-\lambda^{2}(\lambda-1) \\
& =\lambda\left(\lambda^{r-1}-1\right)(\lambda-1)^{3}+\lambda(\lambda-1)\left(\lambda^{2}-3 \lambda+1\right)>P(\mathcal{T}, \lambda)
\end{aligned}
$$


If $\mathcal{H} \cong \mathcal{H}_{6}$, we apply $(2.1)$

$$
\begin{aligned}
P\left(\mathcal{H}_{6}, \lambda\right) & =\left(\lambda^{r}-1\right)(\lambda-1)^{3}+(\lambda-1) \\
& =\lambda\left(\lambda^{r-1}-1\right)(\lambda-1)^{3}+\lambda(\lambda-1)\left(\lambda^{2}-3 \lambda+3\right)>P(\mathcal{T}, \lambda)
\end{aligned}
$$

Thus, $\mathcal{H}$ contains additional edges each of size $r+1$ because $P\left(\mathcal{H}_{i}, \lambda\right)>P(\mathcal{T}, \lambda)$, for $i=1, \ldots, 3, i=5,6$. If we delete these edges in an arbitrary sequence until $\mathcal{H}_{i}$ remains, the order of the hypergraphs resulting by the contraction is always equal 3 . Applying (1.10) repeatedly subtracts from $P\left(\mathcal{H}_{i}, \lambda\right)$ a polynomial of degree 3 . Therefore $P(\mathcal{H}, \lambda)>P(\mathcal{T}, \lambda)$ in each case.

Conversely, if $m \geq 5$ or $m_{2}<m-1$, we can construct a chromatically equivalent hypergraph which is not a hypertree.

Case (1): $\mathcal{H}$ contains two edges of size greater 2.

We can assume that the starting point of our construction is a hyperstar, i.e. all edges have one vertex $u$ in common.

In case of $\mathcal{H} \cong \mathcal{S}_{r, s}, r, s \geq 3$, create $\mathcal{H}_{1}=\left(\mathcal{V}_{1}, \mathcal{E}_{1}\right)$, with $\mathcal{V}_{1}=\mathcal{V} \backslash\left\{v_{e}, v_{f}\right\} \cup\{p, q\}$, $p, q \notin \mathcal{V}$ and with $\mathcal{E}_{1}=\mathcal{E} \backslash\{e, f\} \cup\left\{e_{1}, f_{1}\right\}$, where $e_{1}=e \backslash\left\{v_{e}\right\} \cup\{p\}, f_{1}=f \backslash\left\{v_{f}\right\} \cup\{p\}$. Observe that $e_{1} \nsubseteq f_{1}, f_{1} \nsubseteq e_{1}$, i.e. $e_{1}, f_{1}$ are chromatically active (see Figure 2 ).

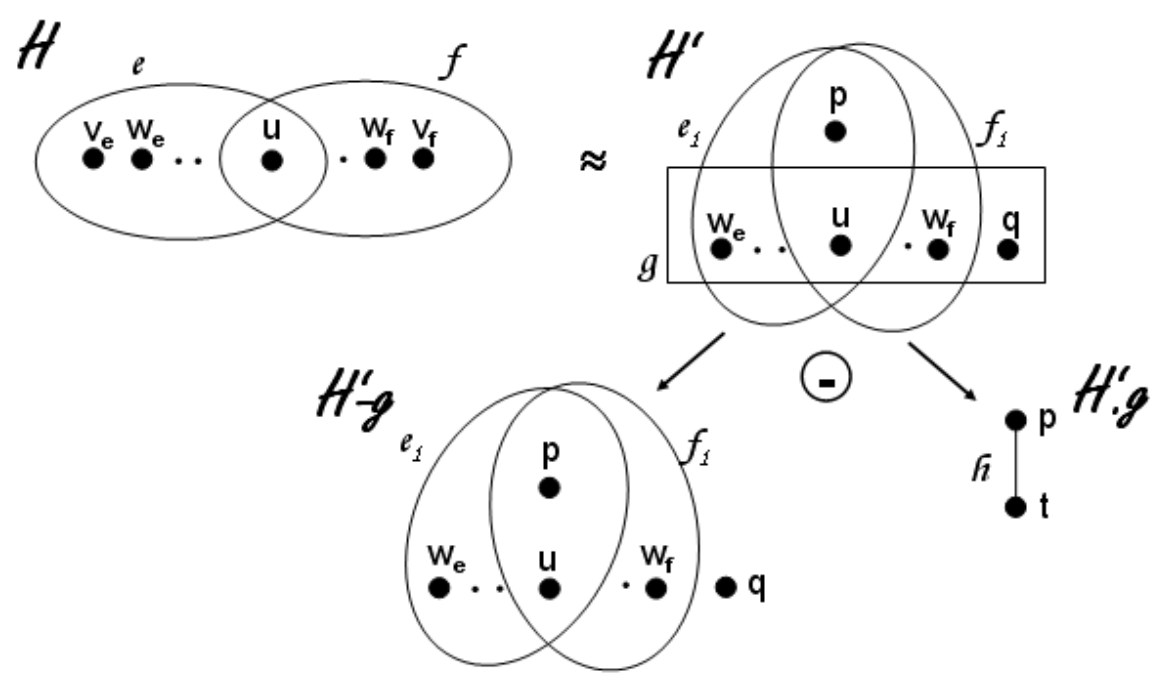

Figure 2

Let $\mathcal{H}^{\prime}=\mathcal{H}_{1}+g$, where $g=e_{1} \cup f_{e} \backslash\{p\} \cup\{q\}$ (see Figure 2). Then $\mathcal{H}^{\prime}-g \cong K_{1} \bullet \mathcal{C}_{r, s}$ and $\mathcal{H}^{\prime} . g \cong K_{2}$.

We apply (1.10)

$$
P\left(\mathcal{H}^{\prime}, \lambda\right)=\lambda\left[\left(\lambda^{r-1}-1\right)\left(\lambda^{s-1}-1\right)+(\lambda-1)\right]-\lambda(\lambda-1)=\lambda\left(\lambda^{r-1}-1\right)\left(\lambda^{s-1}-1\right)
$$


If the hyperstar $\mathcal{H}$ has $m>2$ edges, we take $\mathcal{H}^{\prime \prime} \cong \mathcal{H}^{\prime} \cup_{1} \mathcal{S}$, where $\mathcal{S}$ is the hyperstar defined by the remaining edges. Applying (1.11) to $\mathcal{H}^{\prime \prime}$ completes the proof of this case.

Case (2): If $m \geq 5$, it remains only to consider the cases $m_{2} \geq m-1$.

Let $m=5$. We can assume that $\mathcal{H}$ is of the form given in Figure 3, because (1.8) is independent of the block arrangement of the hypertree. Note that the edge $e$ might be a 2-edge. Then change $\mathcal{H}$ to $K_{1} \cup\left(K_{2} \cup_{1} \mathcal{C}_{(3) 2, r}\right)$.
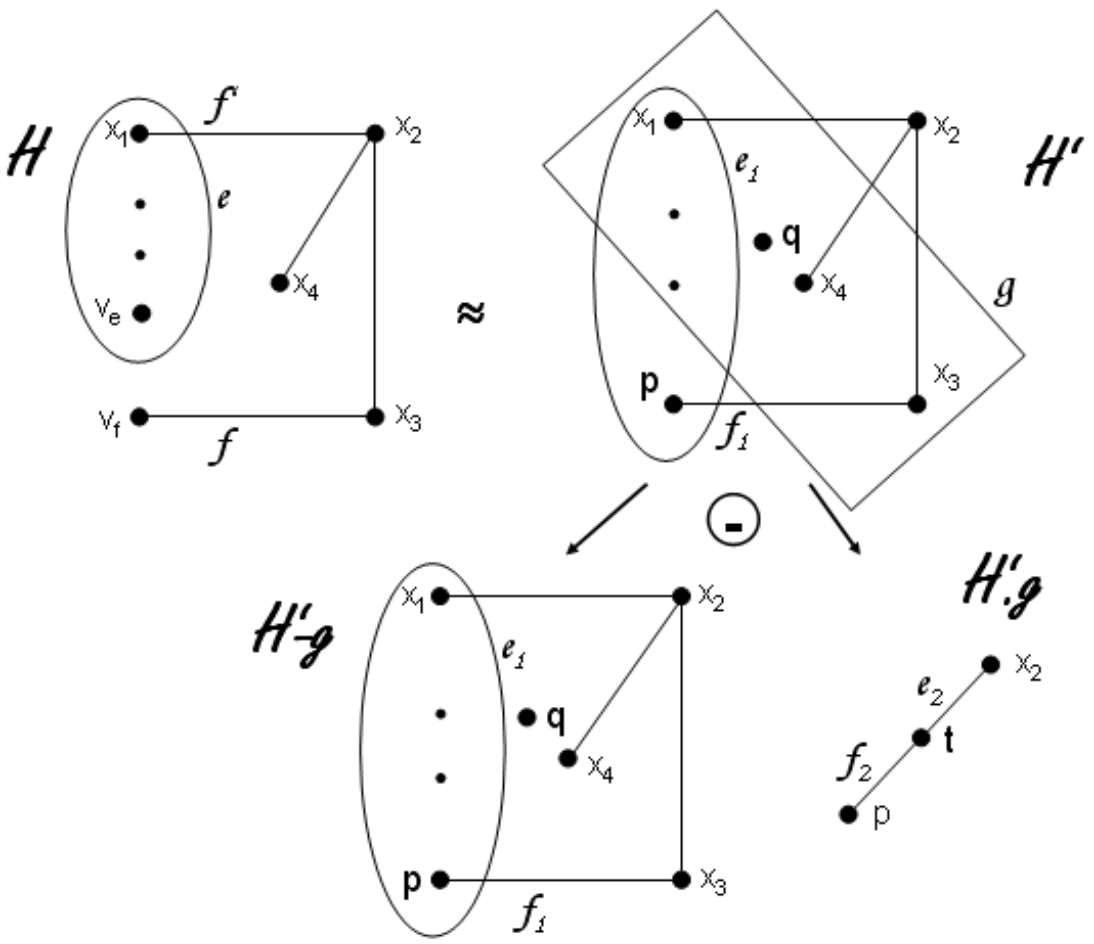

Figure 3

Adding the edge $g=\mathcal{V} \backslash\left\{p, x_{2}\right\}$ yields $\mathcal{H}^{\prime}$. Deleting the edge $g$ yields $\mathcal{H}^{\prime}-g \cong K_{1} \bullet\left(K_{2} \cup_{1} \mathcal{C}_{(3) 2, r}\right)$. Contracting the edge $g$ yields $\mathcal{H}^{\prime} . g \cong \mathcal{S}_{2,2}$.

We apply (1.10)

$$
\begin{aligned}
P\left(\mathcal{H}^{\prime}, \lambda\right) & =\lambda(\lambda-1)\left[(\lambda-1)^{3}\left(\lambda^{r-1}-1\right)+\lambda-1\right]-\lambda(\lambda-1)^{2} \\
& =\lambda(\lambda-1)^{4}\left(\lambda^{r-1}-1\right)+\lambda(\lambda-1)^{2}-\lambda(\lambda-1)^{2}=\lambda(\lambda-1)^{4}\left(\lambda^{r-1}-1\right)
\end{aligned}
$$

If $m>5$, take $\mathcal{H}^{\prime \prime} \cong \mathcal{H}^{\prime} \cup_{1} \mathcal{S}_{(m-5) 2}$. Use of (1.11) completes the proof.

Corollary 3.1. The class of trees with order $n$ is chromatically closed if and only if $n \leq 5$.

\section{Acknowledgments}

The author wishes to thank an anonymous referee for given valuable comments. 


\section{References}

[1] B.D. Acharya, Separability and acyclicity in hypergraphs. Graph theory, Proc. Symp., Calcutta 1976, 1979, pp. 65-83.

[2] J.A. Allagan, A generalization of the chromatic polynomial of a cycle, Comput. Sci. J. Mold. 13 (2005), no. 1, 9-12.

[3] J.A. Allagan, The chromatic polynomials of some linear uniform hypergraphs, Congr. Numerantium 187 (2007), 156-160.

[4] C. Berge, Graphs and hypergraphs, North-Holland, Amsterdam, 1973.

[5] M. Borowiecki and E. Łazuka, Chromatic polynomials of hypergraphs, Discuss. Math., Graph Theory 20 (2000), no. 2, 293-301.

[6] M. Borowiecki and E. Łazuka, On chromaticity of hypergraphs. Discrete Math. 307 (2007), no. 11-12, $1418-1429$.

[7] K. Dohmen, Chromatic polynomials of graphs and hypergraphs. (Chromatische Polynome von Graphen und Hypergraphen.) Düsseldorf: Math.-Naturwiss. Fak., Univ. Düsseldorf, 1993.

[8] F. M. Dong, K. M. Koh, and K. L. Teo, Chromatic polynomials and chromaticity of graphs. Singapore: World Scientific Publishing. xxvii, 2005.

[9] B. Eisenberg, Characterization of a polygonal graph by means of its chromatic polynomial, Proc. $4^{\text {th }}$ southeast. Conf. Comb., Graph Theor., Comput.; Boca Raton 1973, 1973, pp. 275-278.

[10] F. Harary and G. Prins, The block-cutpoint-tree of a graph. Publ. Math. 13 (1966), 103-107.

[11] R.P. Jones, Some results of chromatic hypergraph theory proved by "reduction to graphs", Colloque CNRS, ProblmesCombinatories et Thorie des Graphes 260 (1976), 249-250.

[12] E. Lazuka, On chromaticity of graphs, Discuss. Math., Graph Theory 15 (1995), no. 1, 19-31.

[13] R.C. Read, An introduction to chromatic polynomials, J.Comb.Theory 4 (1968), 52 -71.

[14] M. Sonntag, Antimagic vertex labelings of hypergraphs. Discrete Math. 247 (2002), no. 1-3, 187-199.

[15] M. Sonntag, A characterization of hypercacti. Discrete Math. 307 (2007), no. 21, 2615-2621.

[16] I. Tomescu, Chromatic coefficients of linear uniform hypergraphs, J. Comb. Theory, Ser. B 72 (1998), 229-235.

[17] I. Tomescu, Sunflower hypergraphs are chromatically unique, Discrete Math. 285 (2004), no. 1-3, $355-357$.

[18] I. Tomescu, On the chromaticity of sunflower hypergraphs $S H(n, p, h)$, Discrete Math. 307 (2007), no. $6,781-788$. 